\title{
Responses of a Hodgkin-Huxley Neuron to Various Types of Spike-Train Inputs
}

\author{
Hideo Hasegawa \\ Department of Physics, Tokyo Gakugei University, Koganei, Tokyo 184, Japan
}

(March 28, 2018)

\begin{abstract}
Numerical investigations have been made of responses of a Hodgkin-Huxley (HH) neuron to spike-train inputs whose interspike interval (ISI) is modulated by deterministic, semideterministic (chaotic) and stochastic signals. As deterministic one, we adopt inputs with the time-independent ISI and with time-dependent ISI modulated by sinusoidal signal. The Rössler and Lorentz models are adopted for chaotic modulations of ISI. Stochastic ISI inputs with the Gamma distribution are employed. It is shown that distribution of output ISI data depends not only on the mean of ISIs of spike-train inputs but also on their fluctuations. The distinction of responses to the three kinds of inputs can be made by return maps of input and output ISIs, but not by their histograms. The relation between the variations of input and output ISIs is shown to be different from that of the integrate and fire (IF) model because of the refractory period in the $\mathrm{HH}$ neuron.
\end{abstract}

84.35.+i 87.18.Sn

Typeset using REVTEX 


\section{INTRODUCTION}

Neurons in our brain are known to be responsible for encoding the characteristics of stimuli into a form for further processing by other neurons. During the last decades the anatomical, physiological and theoretical studies on neurons have been extensively made. Despite these efforts, the code used for encoding and decoding in neurons is not clarified at the moment [1]. It is commonly believed that the firing rate reflects the strength of the inputs which trigger the action potentials of neurons. Indeed, the firing activities of motor and sensory neurons vary in response to the applied stimuli. It is not known, however, whether the information is carried through the mean firing rate (rate encoding) or through the details of sequences of the temporarily encoded interspike interval (ISI) (temporal encoding), which is currently controversial [2]- [4]. In the last few years, experimental evidences have accumulated, indicating that many biological systems use the temporal coding. Human visual systems, for example, have shown to classify patterns within $150 \mathrm{msec}$ in spite of the fact that at least ten synaptic stages are involved from retina to the temporal brain [5]. The similar speed of visual processing has been reported for macaque monkeys [6]. Because the firing frequency of neurons involved is less than $100 \mathrm{~Hz}$, each neuron can contribute at most one or two spikes to such computations; there is not sufficient time to sample firing rates.

In recent years, much studies on the encoding of the spike trains by neurons have been made by using the integrate and fire (IF) model [7], which is one of the simplest, dynamical models of neurons $\left[\mathbb{8}\right.$. The IF neutron is silent without the external, input current, $I_{\mathrm{i}}$. When $I_{\mathrm{i}}$, exceeds the critical value, $I_{\mathrm{ic}}$, the IF neuron shows the self-excited oscillations, whose frequency, $f_{\mathrm{o}}$, depends on the magnitudes of $I_{\mathrm{i}}$. It is shown that $f_{\mathrm{o}}$ continuously vanishes when $I_{\mathrm{i}}$ is decreased and approaches to $I_{\mathrm{ic}}$. This behavior of the continuous $f_{\mathrm{o}}-I_{\mathrm{i}}$ dependence is different from the discontinuous one at $I_{\mathrm{ic}}$ in the more realistic Hodgkin-Huxley (HH) neurons [9]; the IF and $\mathrm{HH}$ neurons are classified as the type I and type II, respectively [10]. Furthermore, the IF neuron has the disadvantages of the artificial reset of the action potential and the lack of the refractory time. Although it has been widely employed for the study of neural networks, the IF model is too crude to discuss the activities of real neurons.

The HH model, which well describes the spiking behavior and refractory properties of real neurons, is expressed based on non-linear conductances of $\mathrm{Na}$ and $\mathrm{K}$ ion channels [9]. Since the HH model was proposed, its property has been intensively investigated [11- 117. The behavior of self-excited oscillations of the $\mathrm{HH}$ neuron with the applied current has much variety than that of the IF model. It is shown that the oscillation of the HH neuron may become chaotic when the sinusoidal $I_{\mathrm{i}}$ is applied with proper choices of magnitude and frequency [16] [17]. Such chaotic oscillations are experimentally observed in squid giant axons [14] [15] and Onchidium neurons [18].

The HH model was originally proposed to account for the property of squid giant axons [9] and it has been generalized with modifications of ion conductances [19]. The HH-type models have been widely adopted for a study on activities of transducer neurons such as motor and thalamus relay neurons, which transform the amplitude-modulated input to spike-train outputs. In this paper, we pay our attention to data-processing neurons which receive and emit the spike-train pulses. Assuming that the data-processing neuron may be essentially described by the ion-conductance mechanism of the $\mathrm{HH}$ model, we investigate its inputoutput response in order to get some insight to the following questions: 
(1) How the output ISIs depend on the input ISIs? Does the average rate of the output ISI depend only on the average of the input ISIs?

(2) How do neurons distinguish the different types of deterministic, chaotic and stochastic inputs? How different is the response to different types of spike-train inputs?

Our paper is organized as follows: In the next sec.II, we mention a simple neuron model adopted for our numerical calculation. In sec.III, we investigate the response of our system to deterministic inputs with time-independent ISI (§3.1) and time-dependent ISIs modulated by sinusoidal signal (§3.2). Input and output ISIs are studied by their histograms and return maps; the former shows the distributions and the latter the time correlation of ISI data. In sec.IV, chaotic inputs generated by Rössler (§4.1) and Lorentz model (§4.2) are discussed. Stochastic inputs with the Gamma distribution are treated in sec.V. The final section VI is devoted to conclusion and discussion

\section{ADOPTED MODEL}

We adopt a simple system consisting of a neuron and a synapse. The neuron is assumed to be described by the $\mathrm{HH}$ model and the synapse by the alpha function (Eq.(16)). We will investigate the response of our neuron when spike-train inputs are applied through the synapse.

The HH model is described by the non-linear coupled differential equations for the four variables, $V$ for the membrane potential, and $m, h$ and $n$ for the gating variables of $\mathrm{Na}$ and $\mathrm{K}$ channels, and it is given by [9]

$$
\begin{gathered}
C d V / d t=-g_{\mathrm{Na}} m^{3} h\left(V-V_{\mathrm{Na}}\right)-g_{\mathrm{K}} n^{4}\left(V-V_{\mathrm{K}}\right)-g_{\mathrm{L}}\left(V-V_{\mathrm{L}}\right)+I_{\mathrm{i}}, \\
d m / d t=-\left(a_{m}+b_{m}\right) m+a_{m}, \\
d h / d t=-\left(a_{h}+b_{h}\right) h+a_{h}, \\
d n / d t=-\left(a_{n}+b_{n}\right) n+a_{n},
\end{gathered}
$$

where

$$
\begin{gathered}
a_{m}=0.1(V+40) /\left[1-e^{-(V+40) / 10}\right], \\
b_{m}=4 e^{-(V+65) / 18}, \\
a_{n}=0.01(V+55) /\left[1-e^{-(V+55) / 10}\right], \\
b_{n}=0.125 e^{-(V+65) / 80}, \\
a_{n}=0.07 e^{-(V+65) / 20},
\end{gathered}
$$




$$
b_{n}=1 /\left[1+e^{-(V+35) / 10}\right] .
$$

Here the reversal potentials of $\mathrm{Na}, \mathrm{K}$ channels and leakage are $V_{\mathrm{Na}}=50 \mathrm{mV}, V_{\mathrm{K}}=-77$ $\mathrm{mV}$ and $V_{\mathrm{L}}=-54.5 \mathrm{mV}$; the maximum values of corresponding conductivities are $g_{\mathrm{Na}}=$ $120 \mathrm{mS} / \mathrm{cm}^{2}, g_{\mathrm{K}}=36 \mathrm{mS} / \mathrm{cm}^{2}$ and $g_{\mathrm{L}}=0.3 \mathrm{mS} / \mathrm{cm}^{2}$; the capacity of the membrane is $C=1 \mu \mathrm{F} / \mathrm{cm}^{2}$, details of the $\mathrm{HH}$ model being presented in Refs. [9] [20].

The external, input current, $I_{\mathrm{i}}$, is taken to consist of two terms;

$$
I_{\mathrm{i}}=I_{\mathrm{s}}+I_{\mathrm{p}} .
$$

where $I_{\mathrm{s}}$ expresses the static dc current and $I_{\mathrm{p}}$ denotes the pulse current induced by the spike-train input whose explicit form will be discussed shortly (Eq.(15)).

We consider the delta-function-type spike-train input expressed by

$$
U_{\mathrm{i}}(t)=V_{a} \sum_{n} \delta\left(t-t_{\mathrm{in}}\right) .
$$

The firing time $t_{\text {in }}$ for arbitrary $n$ is assumed to be recurrently defined by

$$
\begin{gathered}
t_{\mathrm{in}+1}=t_{\mathrm{in} n}+T_{\mathrm{in} n}\left(t_{\mathrm{in} n}\right), \\
t_{\mathrm{i} 1}=0,
\end{gathered}
$$

where ISI of input spike, $T_{\mathrm{in}}$, is generally a function of a given time $t_{\mathrm{in} n}$. In this study, we take $T_{\text {in }}$ to be constant ISI, and time-dependent ISI modulated by sinusoidal, chaotic and stochastic signals.

The spike train given by Eq.(12) is assumed to be injected through the synapse, yielding the current $I_{\mathrm{p}}$ given by

$$
I_{\mathrm{p}}(t)=g_{\mathrm{syn}} \sum_{n} \alpha\left(t-t_{\mathrm{in}}\right)\left(V_{a}-V_{\mathrm{syn}}\right) .
$$

Here $g_{\text {syn }}$ and $V_{\text {syn }}$ are the conductivity and potential of synapse, and the alpha function, $\alpha(t)$, is defined by [20]

$$
\alpha(t)=(t / \tau) e^{-t / \tau} \Theta(t),
$$

where $\tau$ is the time constant relevant to the synapse conduction and $\Theta(t)$ is the Heaviside step function. When the ISI is very large compared with $\tau$, Eqs.(15) and (16) yield pulse currents with the maximum value of $I_{\mathrm{p}}^{\max }=e^{-1} g_{\mathrm{syn}}\left(V_{\mathrm{a}}-V_{\mathrm{syn}}\right)$ at $t=t_{\mathrm{in}}+\tau$ and with the half-width of $2.45 \tau$. We assume $V_{a}=30 \mathrm{mV}$ (the typical value of the maximum membrane potential), $V_{\mathrm{syn}}=-50 \mathrm{mV}$ and $\tau=2 \mathrm{msec}$, and treat $g_{\mathrm{syn}}$ as a parameter.

When the membrane potential $V$ oscillates, it yields the spike-train output, which may be expressed by

$$
U_{\mathrm{o}}(t)=V_{a} \sum_{m} \delta\left(t-t_{\mathrm{om}}\right),
$$

in a way similar to Eq.(12), and the output ISI is defined by 


$$
T_{\mathrm{om}}=t_{\mathrm{o} m+1}-t_{\mathrm{om}} .
$$

We will investigate how $T_{\mathrm{om}}$ depends on the various types of $T_{\mathrm{in} n}$.

Differential equations given by Eqs.(1)-(10) including the external current given by Eqs.(11)-(16) are solved by the forth-order Runge-Kutta method for $20 \mathrm{sec}$ with the integration time step of $0.01 \mathrm{msec}$. We discard results of initial ten thousand steps to get stable solutions. If ISI of spike-train input or output is about $10 \mathrm{msec}$, the size of its sample is about 2000. Although this figure is not sufficiently large for statistics of ISI data, we hope an essential ingredient will be clarified in our numerical investigation.

\section{DETERMINISTIC INPUTS}

\subsection{Time-Independent ISI}

\section{A. Pacemaker Neurons}

Let us first consider the $\mathrm{HH}$ neuron without the spike-train input $\left(I_{\mathrm{p}}=0\right)$. The $\mathrm{HH}$ neuron is reported to be silent for $I_{\mathrm{s}}=0$, and to show the self-excited oscillation when $I_{s}$ exceeds the critical value of $I_{\text {ic }}=6.3 \mu \mathrm{A} / \mathrm{cm}^{2}$, above which $T_{\text {on }}$ decreases gradually as $I_{s}$ is increased. The dashed curve in Fig.1(d) expresses an example of the self-excited oscillation with the period of $T_{\mathrm{on}}=10.75 \mathrm{msec}$ for $I_{\mathrm{s}}=25 \mu \mathrm{A} / \mathrm{cm}^{2}$ and $I_{\mathrm{p}}=0$.

Now we apply the spike-train input to this self-excited (pacemaker) neuron. The input is given by Eqs.(10)-(16) with the time-independent ISI of $T_{\mathrm{in}}\left(t_{\mathrm{in}}\right)=T_{\mathrm{i}}=15 \mathrm{msec}$ and $g_{\mathrm{syn}}=0.5 \mathrm{mS} / \mathrm{cm}^{2}$. This spike-train input, $U_{\mathrm{i}}$, shown in Fig.1(a) yields the pulse current, $I_{\mathrm{p}}$, shown in Fig.1(c), by which the membrane potential, $V$, oscillates as depicted by the solid curve in Fig.1(d). We plot in Fig.1(b) the time sequence of the spike-train output, $U_{\mathrm{o}}$, which should be compared with the input $U_{\mathrm{i}}$. The pulse current, $I_{\mathrm{p}}$, has the maximum value of $I_{\mathrm{p}}^{\max }=14.8 \mu \mathrm{A} / \mathrm{cm}^{2}$ at $t=t_{\mathrm{in}}+\tau$ msec. We notice that the oscillation in $V$ is rather different from that shown by the dashed curve for $I_{\mathrm{p}}=0$. Figure 2 expresses the histogram of the output ISI, showing that $\left\{T_{0}\right\}$ distributes continuously between 8.36 to 11.62 msec. The mean and root-mean-square (rms) values of the output ISI are $\mu_{\mathrm{o}}=10.43$ and $\sigma_{\mathrm{o}}=1.12 \mathrm{msec}$. respectively. This oscillation is chaotic as was pointed out for the $\mathrm{HH}$ neuron receiving sinusoidal inputs [16] [17]; the pulse current $I_{\mathrm{p}}$ shown in Fig.1(c) is not so different from the sinusoidal one in a crude sense. The chaotic behavior is clearly seen in Fig.3, which depicts return maps of input and output ISIs.

When the ISI value of spike-train input, $T_{\mathrm{i}}\left(=\mu_{\mathrm{i}}\right)$, is changed, we get an interesting behavior in $\mu_{o}$ as shown in Fig.4, where the solid (dashed) curve expresses $\mu_{\mathrm{o}}\left(\sigma_{\mathrm{o}}\right)$, and filled circles express the distribution of $\left\{T_{\mathrm{on}}\right\}$ for a given $\mu_{\mathrm{i}}$. We note that for $\mu_{\mathrm{i}}=9-11$ msec, the period of the oscillation is forced to be the same; $T_{\mathrm{om}}=T_{\mathrm{i}}$, leading to the ratio: $k \equiv \mu_{\mathrm{o}} / \mu_{\mathrm{i}}=1$. When $\mu_{\mathrm{i}}=5 \mathrm{msec}$, we get $\mu_{o}=10 \mathrm{msec}$ and then $k=2$. On the contrary, for $\mu_{\mathrm{i}}=20 \mathrm{msec}$, we get the two values of $T_{\mathrm{on}}=9$ and $11 \mathrm{msec}$, and $\mu_{\mathrm{o}}=10$, the average period of the output being a half of the input $(k=1 / 2)$. This is also the case for $\mu_{\mathrm{i}}=21$ and $22 \mathrm{msec}$. In the other cases noticed above, the ISI of output distributes between about $8.5-11.5 \mathrm{msec}$. We should note that irrespective of $\mu_{\mathrm{i}}$, output ISI is always about $10 \mathrm{msec}$, which is nearly equal to $T_{\mathrm{o}}=10.75 \mathrm{msec}$, ISI for $I_{\mathrm{s}}=25$ and $I_{\mathrm{p}}=0 \mu \mathrm{A} / \mathrm{cm}^{2}$. 


\section{B. Silent Neurons}

Next consider the silent neuron with $I_{\mathrm{s}}=0$, for which the oscillation of the membrane potential is induced by applied spike-train inputs. Figures 5(a)-(d) show the calculated result in which the spike-train input is given by $T_{\mathrm{in}}=\mu_{\mathrm{i}}=10 \mathrm{msec}$ and $g_{\mathrm{syn}}=0.5 \mathrm{mS} / \mathrm{cm}^{2}$ without static currents $\left(I_{\mathrm{s}}=0\right)$. The applied spike-train inputs shown in Fig.5(a) create the pulse current with the peaks of $I_{\mathrm{p}}^{\max }=15.3 \mu \mathrm{A} / \mathrm{cm}^{2}$ as shown in Fig.5(c). The induced oscillation of the membrane potential, $V$, in Fig.5(d) is phase locked with the ratio of $4: 3$, oscillating with a long cycle of $40.00 \mathrm{msec}(=11.25+12.36+16.39)=4 \mu_{i}$, where 11.25 , 12.36 and 16.39 are the values of output ISIs. The return map of output ISIs is plotted in Fig.6(a).

Figure 7 shows $\mu_{o}$ and $\sigma_{\mathrm{o}}$ as a function of $\mu_{\mathrm{i}}$. We notice that $\mu_{\mathrm{o}}$ agrees with $\mu_{\mathrm{i}}(k=1)$ for $\mu_{\mathrm{i}}$ greater than $12 \mathrm{msec}$, where the $\mathrm{HH}$ neuron behaves as a simple transmitter with a delay of about $2.0 \mathrm{msec}$. This is in strong contrast with the behavior of the pacemaker neuron discussed in the preceding sub-section (Fig.4). On the other hand, for $\mu_{\mathrm{i}}$ less than 11 msec, the behavior of output ISI is rather complicated. It is easy to see that $k=2$ for $\mu_{\mathrm{i}}=6,7$ and $8 \mathrm{msec}$, and that $k=3$ for $\mu_{\mathrm{i}}=4 \mathrm{msec}$. For $\mu_{\mathrm{i}}=9$, we get $T_{\mathrm{om}}=12.06$ and $14.96 \mathrm{msec}$, leading to a longer period of $3 \mu_{i}=27.00(=12.06+14.96)$ msec. For $\mu_{\mathrm{i}}=5 \mathrm{msec}$, we get $T_{\mathrm{om}}=10.94$ and $14.06 \mathrm{msec}$, which leads to a long period of $5 \mu_{\mathrm{i}}=25 \mathrm{msec}$, its return map being shown in Fig.6(b). Surprisingly, a much longer period of $13 \mu_{\mathrm{i}}$ is realized for $\mu_{\mathrm{i}}=11$ msec. The rms value of $\sigma_{\mathrm{o}}$ has an appreciable value only around $\mu_{\mathrm{i}}=10 \mathrm{msec}$.

We have repeated our calculation by changing the value of $g_{\text {syn }}$. The calculated ratio, $k=\mu_{\mathrm{o}} / \mu_{\mathrm{i}}$, is shown as functions of $g_{\mathrm{syn}}$ and $\mu_{\mathrm{i}}$ in Fig.8, where only the integer values of $k$ are shown by circles $(k=1)$, squares $(k=2)$, triangles $(k=3)$ and diamond $(k=4)$. Note that non-integer values of $k$ exist between the integer values; for example, $k=4 / 3$ for $g_{\text {syn }}=0.5 \mathrm{mS} / \mathrm{cm}^{2}$ and $\mu_{\mathrm{i}}=10 \mathrm{msec}$ (Fig.7). We cannot obtain spike-train outputs for small synaptic couplings as expected. When $\mu_{\mathrm{i}}=10 \mathrm{msec}$, we get the critical value of $g_{\text {syn }}=0.11 \mathrm{mS} / \mathrm{cm}^{2}$ below which no outputs are available. This coupling yields the pulse current with the maximum value of $I_{\mathrm{p}}^{\max }=1.6 \mu \mathrm{A} / \mathrm{cm}^{2}$, which is much smaller than the critical dc current of $I_{\text {ic }}=6.3 \mu \mathrm{A} / \mathrm{cm}^{2}$ for the self-excited oscillation with $I_{\mathrm{p}}=0$. We note that we get $k=1$ for the large ISIs with fairly strong synaptic couplings. When we decrease $\mu_{\mathrm{i}}$ with keeping $g_{\mathrm{syn}}$ fixed, values of $k$ become larger since the $\mathrm{HH}$ neuron cannot respond to inputs with the small ISI because of its refractory period. Figure 8 reminds us the result of Guttman, Feldman and Jakobson [15] who reported in their Table 1, the calculated $k$ as functions of the magnitude, $A$, and the frequency, $f_{\mathrm{i}}$, when the sinusoidal input given by $I_{\mathrm{i}}=A \sin \left(2 \pi f_{\mathrm{i}}\right)+I_{\mathrm{b}}$ is applied to squid giant axons with a bias current, $I_{\mathrm{b}}$. Our result for

$\mu_{\mathrm{i}}<10$ msec fairly agrees with that of Ref. [15]. However, the agreement between the two results is not good for $\mu_{\mathrm{i}} \gg 10 \mathrm{msec}$, where our input current with the pulse width of about $2.45 \tau \sim 5$ msec (Eqs.(15) and (16)) is quite different from the sinusoidal current adopted in Ref. 15.

As was shown in Fig.4, the pacemaker HH neuron emits the output ISI of $T_{\mathrm{om}} \sim 10$ msec irrespective of the value of input ISI, and then it is considered to be inadequate as a data processor. Then, in the following sections, we will investigate only the silent $\mathrm{HH}$ neuron with a fixed value of $g_{\text {syn }}=0.5 \mathrm{mS} / \mathrm{cm}^{2}$.

\subsection{ISI with Sinusoidal Modulation}


In this sub-section we discuss an application of the spike-train input whose ISI is modulated by the sinusoidal signal given by

$$
T_{\mathrm{i} n}(t)=d_{0}+d_{1} \sin \left(2 \pi t / T_{p}\right),
$$

where $T_{\mathrm{p}}$ is the period and $d_{0}$ and $d_{1}$ are coefficients adjusting $\mu_{\mathrm{i}}$ and $\sigma_{\mathrm{i}}$.

Figure $9(\mathrm{a})$ and (b) show the time course of input $U_{\mathrm{i}}$ and output $U_{\mathrm{o}}$ for $d_{0}=2 d_{1}=$ $20 \mathrm{msec}$ with $T_{p}=100 \mathrm{msec}$. Because of the introduced sinusoidal modulation, ISIs at $100<t<150 \mathrm{msec}$ are, for example, larger that those at $150<t<200 \mathrm{msec}$. Figure 9 (c) depicts the return map of input ISIs, which has the egg-shape circle expected for the sinusoidal signal. On the other hand, the return map of output ISIs shown in Fig.9(d) reveals the chaotic behavior. Results for $d_{0}=2 d_{1}=10$ are plotted in Fig.10(a)-(d), which show that although the return map of input ISI has the egg-shape circle, that of output ISIs is distorted. The reason of this distortion is explained in Fig.11, where solid histograms express input and output ISIs for $d_{0}=2 d_{1}=10(\mu=8.68, \sigma=3.42)$ and dashed histograms those for $d_{0}=2 d_{1}=20\left(\mu_{i}=17.54, \sigma_{i}=6.94\right)$, with $T_{p}=100 \mathrm{msec}$. (It is noted that we get $\mu_{i}<d_{0}$ because the histogram of the input ISI at $T_{\mathrm{i} n}<d_{0}$ has larger magnitudes than that at $T_{\text {in }}>d_{0}$ ). In the case of $d_{0}=2 d_{1}=20 \mathrm{msec}$, the input and output ISIs distribute almost in the same region at $11<T_{\mathrm{om}}<30 \mathrm{msec}$. On the contrary, in the case of $d_{0}=2 d_{1}=10 \mathrm{msec}$, the output ISIs distribute at $11.01<T_{\mathrm{om}}<19.48 \mathrm{msec}$ while input ISIs are at $5.00<T_{\mathrm{i} n}<14.96 \mathrm{msec} ;$ no output ISIs at $T_{\mathrm{o} m}<11 \mathrm{msec}$. This is due to the refractory period of the $\mathrm{HH}$ neuron and it is the origin of the distortion in the return map shown in Fig.10(b). Defining the dimensionless coefficients of variations for input and output ISIs by

$$
c_{\mathrm{v} \lambda}=\sigma_{\lambda} / \mu_{\lambda}, \quad(\lambda=\mathrm{i} \text { and } \mathrm{o})
$$

we get $c_{\mathrm{vo}}=0.17$ and 0.38 for $d_{0}=2 d_{1}=10$ and 20 msec, respectively; note that $c_{\mathrm{vi}}=0.40$ for both inputs.

Figure 12 shows $\mu_{\mathrm{o}}$ and $\sigma_{0}$ calculated by changing $\mu_{\mathrm{i}}$ with the fixed value of $c_{\mathrm{vi}}=0.40$. Solid and dashed curves denote $\mu_{\mathrm{o}}$ and $\sigma_{\mathrm{o}}$, respectively, and filled circles the distribution of $\left\{T_{\mathrm{om}}\right\}$ for a given $\mu_{\mathrm{i}}$. We notice that there is no output ISIs with $T_{\mathrm{om}}$ less than about 10 msec, which shows characteristic of the low-pass filter of the silent HH neuron .

\section{CHAOTIC INPUTS}

\subsection{Rössler Model}

In this section, we study the spike-train input whose ISI is modulated by chaotic signals. First we adopt the Rössler model, which is given by

$$
\begin{gathered}
d x / d t=-y-z, \\
d y / d t=x+a y, \\
d z / d t=b x-c z+x z,
\end{gathered}
$$


with $a=0.36, b=0.4$ and $c=4.5$ [21]. Since ISI has to be positive and the characteristic time scale in the Rössler model is different from that of the $\mathrm{HH}$ model, we adopt the variable $x(t)$ which yields

$$
T_{\mathrm{in} n}\left(t_{\mathrm{in}}\right)=d_{0}+\left(d_{1} / 10\right) x\left(p t_{\mathrm{in}}\right),
$$

with the following two choices of parameters:

$$
\begin{array}{ll}
d_{0}=d_{1}=10 \mathrm{msec} \text { and } p=1 / 10 & \text { (case R1), } \\
d_{0}=d_{1}=20 \mathrm{msec} \text { and } p=1 / 20 & \text { (case R2). }
\end{array}
$$

Figure 13(a) and (b) show the time course of input and output spike trains for the case R1. The return map of input ISI depicted in Fig.13(c) shows a shape characteristic for chaotic signals. On the other hand, the return map of output ISIs is rather strange with no traces at $T_{\mathrm{o}}<10 \mathrm{msec}$. This is due to the low-pass filter behavior of the silent $\mathrm{HH}$ neuron, as shown by solid histograms in Fig.14(a) and (b); output ISIs distribute at $11.11<T_{\mathrm{om}}<25.15 \mathrm{msec}\left(\mu_{\mathrm{om}}=13.43, \sigma_{\mathrm{o}}=2.44 \mathrm{msec}\right)$ whereas input ISIs distribute at $5.06<T_{\text {in }}<16.56 \mathrm{msec}\left(\mu_{\mathrm{i}}=9.53, \sigma_{\mathrm{i}}=2.69 \mathrm{msec}\right)$.

Return maps for the case R2 are shown in Fig.15, in which both return maps are almost the same. This is because input and output ISIs locate almost in the same region of $10<$ $T_{\mathrm{i} n}, T_{\mathrm{om}}<30 \mathrm{msec}$, as shown by dashed histograms in Fig.14(a) and (b).

Next we investigate the nature of the correlation in the ISI sequences. This is made by employing the surrogate data method applied to ISI data [23]. We adopt the shuffled surrogate as a simple method to get surrogate data. The distributions of ISIs of shuffled surrogate inputs are exactly the same as those of original ISI data although surrogate data have no correlation between successive ISI values.

The time course of the membrane potentials for the surrogate data is ostensibly quite similar to that for the original chaotic input (not shown). The solid (dashed) histogram in Fig.14(c) shows the distribution of output ISIs of surrogate data generated from the Rössler model for the case R1 (R2). The results of the surrogate data are similar to those for the corresponding original data. Return maps of the input and output ISI of the surrogate data, depicted in Fig.15(c) and (d), show the characteristics of random signals.

\subsection{Lorentz Model}

The similar calculation is made with the use of the Lorentz model, which is given by

$$
\begin{gathered}
d x / d t=d(y-x), \\
d y / d t=e x-y-x z, \\
d z / d t=-f z+x y,
\end{gathered}
$$

with $d=10, e=28$ and $f=8 / 3$ 22]. We employ the variable $z(t)$, with which the input ISI is given by

$$
T_{\mathrm{in}}\left(t_{n}\right)=d_{0}+\left(d_{1} / 25\right)\left(z\left(p t_{\mathrm{in}}\right)-25\right),
$$


where $d_{0}=d_{1}=20$ and $p=1 / 100$.

Figures 16(a)-(d) show return maps of ISI data of original chaotic data and its surrogate. Return maps of output ISIs for the chaotic and surrogate data shown in Fig.16(b) and (d) have no traces at $T_{\mathrm{om}}>10 \mathrm{msec}$ because of the low-pass filter character of the $\mathrm{HH}$ neuron.

Sauer [24], and Racicot and Longtin [25] studied the response of the IF model to the input whose amplitudes are modulated by chaotic signals. It was shown that when the mean firing rate is high, the relationship between input and output is high, which leads to the high nonlinear predicability. Our calculations have shown that when the mean firing rate is too high (i.e. input ISIs are too short such as $T_{\mathrm{i} n}<10 \mathrm{msec}$ ), the information is lost because the $\mathrm{HH}$ neuron behaves as the low-pass filter due to its refractory period, which is not included in the IF model.

\section{STOCHASTIC INPUTS}

The ISIs of spike-train input, $T_{\mathrm{i} n}$, in Eq.(13) are assumed to be independent random variables with the Gamma probability density function given by

$$
P(T)=s^{r} T^{r-1} e^{-s T} / \Gamma(r)
$$

for which we get $\mu_{\mathrm{i}}=r / s, \sigma_{\mathrm{i}}=\sqrt{r} / s$ and $c_{v i}=1 / \sqrt{r}, \Gamma(r)$ being the gamma function. It is noted that in the limit of $r=1$, Eq.(29) reduces to the Poisson distribution $\left(c_{\mathrm{vi}}=1\right)$ and that in the limits of $r \rightarrow \infty$ and $s \rightarrow \infty$ with keeping $\mu_{\mathrm{i}}=r / s$ fixed, Eq.(29) reduces to $P(T)=\delta\left(T-\mu_{\mathrm{i}}\right)$, the constant ISI with $\mu_{\mathrm{i}}=T$ and $c_{\mathrm{vi}}=0$.

The spike-train input created by the Gamma-distribution generator is applied to our neural system. Calculations are performed by changing $\mu_{\mathrm{i}}$ by keeping the value of $c_{\mathrm{vi}}$ fixed. Note that because the number of our sample of input ISI is not sufficiently large, the obtained $c_{\mathrm{vi}}$ fluctuates around the intended values. Solid histograms in Fig.17(a) and (b) show the result for $c_{\mathrm{vi}}=0.40, \mu_{\mathrm{i}}=10 \mathrm{msec}, c_{\mathrm{vo}}=0.25$ and $\mu_{\mathrm{o}}=14.84 \mathrm{msec}$ while dashed histograms for the result for $c_{\mathrm{vi}}=0.40, \mu_{\mathrm{i}}=20 \mathrm{msec}, c_{\mathrm{vo}}=0.36$ and $\mu_{\mathrm{o}}=21.11 \mathrm{msec}$.

Solid and dashed curves in Fig.18(a) denote $\mu_{\mathrm{o}}$ and $\sigma_{\mathrm{o}}$, respectively, for $c_{\mathrm{vi}}=0.4$. We note that as increasing $\mu_{\mathrm{i}}, \mu_{\mathrm{o}}$ increases and approaches the dotted line expressing $\mu_{\mathrm{o}}=\mu_{\mathrm{i}}$. This is similar to the case of $c_{\mathrm{vi}}=0$ shown in Fig.7, where $\mu_{\mathrm{o}}=\mu_{\mathrm{i}}$ at $\mu_{\mathrm{i}} \gtrsim 10 \mathrm{msec}$. On the contrary, the dependence of $\mu_{\mathrm{o}}$ on $\mu_{\mathrm{i}}$ for the case of $c_{\mathrm{vi}}=1.0$ shown in Fig.18(b), is rather different from the cases of $c_{\mathrm{vi}}=0$ and 0.4 . We get $\mu_{\mathrm{o}} \sim\left(\mu_{\mathrm{i}}+10\right) \mathrm{msec}$ at $\mu_{\mathrm{o}}<100 \mathrm{msec}$ and it deviates from the dotted line showing $\mu_{\mathrm{o}}=\mu_{\mathrm{i}}$. These calculations depicted in Figs.18(a) and (b) clearly show that $\mu_{\mathrm{o}}$ depends not only on $\mu_{\mathrm{i}}$ but also on $\sigma_{\mathrm{i}}\left(c_{\mathrm{vi}}\right)$. Although both $\mu_{\mathrm{o}}$ and $\sigma_{\mathrm{o}}$ increase as the value of $\mu_{\mathrm{i}}$ is increased, the increase in the latter is more significant than that in the former, which yields an increase in $c_{\mathrm{vo}}$, as shown by the thin-solid curves in Figs.18(a) and (b). We note that $c_{\mathrm{vo}}$ approaches the values of $c_{\mathrm{vi}}$ as increasing $\mu_{\mathrm{i}}$, the related discussion being given in Sec.VI.

We have performed the calculation also using input ISI with random, uniform distribution. Obtained results are similar to those for the Gamma distribution, as far as the adopted values of $c_{\mathrm{vi}}$ are the same (not shown).

\section{CONCLUSION AND DISCUSSION}


We have investigated the responses of the $\mathrm{HH}$ neurons, by applying the various types of spike-train inputs whose ISI is modulated by deterministic, chaotic and stochastic signals. The obtained results are summarized as follows:

(1) Output ISIs of the pacemaker HH neuron against the time-independent input ISI are always $T_{\mathrm{o} m} \sim 10 \mathrm{msec}$ irrespective of the value of the input ISI (Fig.4).

(2) Output ISIs of the silent HH neurons for the constant ISI with $T_{\mathrm{in}}>10$ msec yield output ISI with $T_{\mathrm{om}}=T_{\mathrm{i} n}$ whereas for ISI with $T_{\mathrm{i} n}<10 \mathrm{msec}$, the HH neuron generally emits multiple numbers of output ISIs (Fig.7).

(3) For the input ISI modulated by sinusoidal, chaotic and stochastic signals, the silent $\mathrm{HH}$ neuron behaves as a low-pass filter because of its refractory period, yielding output ISI with $T_{\mathrm{om}}>10 \mathrm{msec}$.

(4) Output ISIs generally depend not only on the mean of the input ISI but also on their fluctuations: the $\mathrm{HH}$ neuron is not a simple integrator.

(5) The analysis on the histograms of input and output ISIs cannot distinguish the responses to the deterministic, chaotic and stochastic signals.

(6) The distinction can be made by an analysis of the time correlation of the ISI data, for example, by plotting their return maps.

Softky and Koch [26] have reported a large coefficient of variation $\left(c_{\mathrm{vo}}=0.5 \sim 1.0\right)$ for spike trains of non-bursting cortical neurons in visual V1 and MT of monkeys in strong contrast with a small $c_{\mathrm{vo}}(=0.05 \sim 0.1)$ in motor neurons [27]. In order to explain the large $c_{\mathrm{vo}}$, several hypotheses have been proposed; a balance between excitatory and inhibitory inputs [28, the high physiological gain in the $f_{\mathrm{o}}-I_{\mathrm{i}}$ plot [29], correlation fluctuations in recurrent networks [30], and the active dendrite conductance [31]. By using the IF model, Feng and Brown [32] have shown that there are three kinds of behaviors of $c_{\mathrm{vo}}$ depending on the distribution of input ISIs;

(a) $c_{\mathrm{vo}}$ tends to decrease for the Gaussian, uniform or truncated distribution of ISIs,

(b) $c_{\mathrm{vo}}$ remains constant for the exponentially distributed ISIs, and

(c) $c_{\mathrm{vo}}$ diverges to infinity when ISIs follow the Pareto distribution which has a slowdecreasing tail of $T^{-\alpha}(\alpha>0)$ at large $T$.

The case (a) was previously discussed by Marsalek, Koch and Maunsell [33].

Figure 19 shows the dependence of $c_{\mathrm{vo}}$ on $c_{\mathrm{vi}}$ for various types of our input ISIs having been reported in previous sections. Inverted triangles denote the results for the constant ISIs (§3.1B), open marks the results for input ISIs with sinusoidal modulation (§ IV), and filled circles, triangles and squares results of the stochastic modulation $(\S \mathrm{V})$. Our calculations show the followings:

(i) The constant ISI with a vanishing $c_{\mathrm{vi}}$ yields the finite $c_{\mathrm{vo}}(<0.2)$, i.e. $\quad c_{\mathrm{vo}} \gtrsim c_{\mathrm{vi}}$,

(ii) the finite-width distribution of ISIs with the sinusoidal modulation leads to $c_{\mathrm{vo}} \lesssim c_{\mathrm{vi}}$, and

(iii) the exponential, Gamma distribution of input ISIs yield the result which is ostensibly similar to that in the item (ii).

Although the item (ii) is in agreement with the result for the above-mentioned case (a) discussed in Refs. [32] and [33], the items (i) and (iii) disagree with the results of the cases of (a) and (b), respectively. This difference is expected to arise from the fact that the response of the HH neuron with the refractory period is different from that of the IF neuron without it [10]. 
Finally we want to discuss the transient response of the $\mathrm{HH}$ neuron to the cluster of spike-train inputs. Figure 20(a), (b), and (c) show the results for $T_{\mathrm{i} n}=5,10$ and $20 \mathrm{msec}$, respectively. In Fig.20(c), for example, the upper (lower) panel of C1, C2, C3 and C4 express the time courses of input (output) spike trains for inputs of two, three, four and five impulses, respectively, with $T_{\mathrm{i} n}=20 \mathrm{msec}$. In this case the ISI of output pulses is the same as that of input pulses, $T_{\mathrm{om}}=T_{\mathrm{i} n}$, because the $\mathrm{HH}$ neuron behaves as a linear transmitter

for inputs with ISI of $T_{\mathrm{i} n} \gtrsim 10 \mathrm{msec}$. On the contrary, its behaviors of output ISI data for inputs with $T_{\mathrm{in}}=5$ and $10 \mathrm{msec}$ are much complicated. Fig.20(a) shows that output ISIs for $T_{\mathrm{i} n}=5 \mathrm{msec}$ are 11.39 and $11.87 \mathrm{msec}$, which should be compared with $T_{\mathrm{om}}=10.94$ and $14.06 \mathrm{msec}$ for the sequence of the spike trains with constant ISI of 5 msec discussed in sec. 3.1B. For the case of $T_{\mathrm{in}}=10 \mathrm{msec}$ shown in Fig.20(b), we get $T_{\mathrm{om}}=11.44,11.80$ and 17.11 msec whereas the sequence of the constant ISI of 10 msec leads to $T_{\text {om }}=11.25,12.36$ and 16.39 msec (Fig.5). It is noted that both inputs with three (A2) and four impulses (A3) yield the same output of two impulses. Similarly, inputs with three (B2) and four impulses (B3) lead to outputs with three impulses. We should note in all the cases shown in Fig.20(a)-(c) that the first output pulse is rather quickly emitted with a delay of $2.1 \mathrm{msec}$ after the first input pulse of clusters is applied to the $\mathrm{HH}$ neuron. This fast transient response may be relevant to a quick passage of information reported by Thorpe, Eize and Marlot [5] and by Rolls and Tovee [6].

\section{ACKNOWLEDGEMENTS}

This work is partly supported by a Grant-in-Aid for Scientific Research from the Japanese Ministry of Education, Science and Culture. 


\section{REFERENCES}

[1] F. Rieke, D. Warland, R. Steveninck, and W. Bialek, Exploring the Neural Code (MIT press, England, 1996).

[2] T. J. Sejnowski, Nature 376, 21 (1995).

[3] J. J. Hopfield, Nature 376, 33 (1995).

[4] D. Ferster and N. Spruston, Science 270, 756 (1995).

[5] S. Thorpe, D. Fize and C. Marlot, Nature 381, 520 (1996).

[6] E. T. Rolls and M. J. Tovee, Proc. Roy. Soc. B 257 9, (1994)

[7] W. Maass, Neural Comput. 9, 279 (1997); related references are therein.

[8] For a review on neuron models see, W. Gerstner, Phys. Rev. E 51, 738 (1995).

[9] A. L. Hodgkin and A. F. Huxley, J. Physiol. 117, 500 (1952).

[10] B. S. Gutkin, G. B. Ermentrout, Neural Computation 10, 1047 (1098).

[11] I. Nemoto, S. Miyazaki, M. Saito, T. Utsunomiya, Biophys. J. 15, 469 (1976).

[12] A. V. Holden, Biol. Cybernetics 21, 1 (1976).

[13] J. F. Fohlmeister, W. J. Adelman, and R. E. Poppele, Biophys. J. 30, 79 (1980).

[14] G. Matsumoto, K. Kim, T. Ueda, and J. Shimada, J. Phys. Soc. Jpn. 49, 906 (1980).

[15] R. Guttman, L. Feldman, and E. Jakobsson, J. Memb. Biol. 56, 9 (1980).

[16] K. Aihara, G. Matsumoto, and Y. Ikegaya, J. Theor. Biol. 109, 249 (1984).

[17] G. Matsumoto, K. Aihara, M. Ichikawa, and A. Tasaki, J. Theor. Neurobiol. 3, 1 (1984).

[18] H. Hayashi, S. Ishizuka and K. Hirakawa, J. Phys. Soc. Jpn. 54, 2337 (1985).

[19] C. Koch and Ö. Bernander, The Handbook of Brain Theory and Neural Networks, ed. M. A. Arbib (MIT press, England, 1995), p129-134.

[20] M. Park and S. Kim, J. Korean Phys. Soc. 29, 9 (1996).

[21] O. E. Rössler, Phys. Lett. 57A, 397 (1976).

[22] E. Lorentz, J. Atmos. Sci. 20, 131 (1963).

[23] J. Theiler, S. Eubank, A. Longtin, B. Galdrikian and J. D. Farmer, Physica D 58, 77 (1992).

[24] T. Sauer, Phys. Rev. Lett. 72, 3811 (1994).

[25] D. M. Racicot, and A Longtin, Physica D 104, 184 (1997).

[26] W. R.Softky and C. Koch, Neural Comput. 4, 643 (1992).

[27] W. Calvin and C. Stevens, J. Neurophys. 31, 574 (1968).

[28] M. Shadlen and W. T. Newsome, Curr. Opin. Neurobiol. 4, 569 (1994).

[29] T. W. Troyer, and K. D. Miller, Neural Computation 10, 1047 (1098).

[30] M. Usher, M. Stemmer, C. Koch and Z. Olami, Neural Comp. 6, 795 (1994).

[31] W. R.Softky, Curr. Opin. Neurobiol. 5, 239 (1995).

[32] J. Feng and D Brown, J. Phys. A 31, 1239 (1998).

[33] P. Marsalek, C. Koch and J. Maunsell, Proc. Natl. Acad. Sci. USA 94, 735 (1997). 


\section{FIGURES}

FIG. 1. Responses of the pacemaker HH neuron to the time-independent input ISI ( $I_{\mathrm{p}}$ with $T_{\mathrm{i}}=15 \mathrm{msec}$ and $I_{\mathrm{s}}=25 \mu \mathrm{A} / \mathrm{cm}^{2}$; time sequences of (a) input $U_{\mathrm{i}}$, (b) output $U_{\mathrm{o}}$, (c) pulse current $I_{\mathrm{p}}$, and (d) membrane potential $V$, dashed curves in (b) and (d) denoting the result with $I_{\mathrm{p}}=0$.

FIG. 2. Histograms of (a) the time-independent input ISI $\left(T_{i}=15 \mathrm{msec}\right)$ and (b) output ISI of the pacemaker neuron (see Fig. 1).

FIG. 3. Return maps of (a) time-independent input ISI with $T_{\mathrm{i}}=15 \mathrm{msec}$ and (b) output ISI of the pacemaker neuron (Fig.1).

FIG. 4. Mean ( $\mu_{\mathrm{o}}$, solid curve) and rms ( $\sigma_{\mathrm{o}}$, dashed curve) values of output ISI of pacemaker neurons $\left(I_{\mathrm{S}}=25 \mu \mathrm{A} / \mathrm{cm}^{2}\right)$ against the mean value $\left(\mu_{\mathrm{i}}\right)$ of time-independent input ISI. Filled circles denote the distribution of output ISIs for a given $\mu_{\mathrm{i}}$, dotted curves denoting $k \equiv \mu_{\mathrm{o}} / \mu_{\mathrm{i}}$.

FIG. 5. Response of the silent HH neuron to the time-independent input ISI ( $I_{\mathrm{p}}$ with $T_{\mathrm{i}}=10$ msec and $I_{\mathrm{s}}=0$ ); time sequences of (a) the input $U_{\mathrm{i}}$, (b) output $U_{\mathrm{o}}$, (c) pulse current $I_{\mathrm{p}}$, and (d) membrane potential $V$.

FIG. 6. Return maps of output ISIs for the time-independent input with (a) $T_{\mathrm{i}}=10$ and (b) 5 msec (see Fig.5).

FIG. 7. Mean ( $\mu_{\mathrm{o}}$, solid curve) and $\mathrm{rms}\left(\sigma_{\mathrm{o}}\right.$, dashed curve) values of output ISI of silent neurons against the mean value $\left(\mu_{\mathrm{i}}\right)$ of time-independent input ISI. Filled circles denote the distribution of output ISIs for a given $\mu_{\mathrm{i}}$, dotted curves denoting $k \equiv \mu_{\mathrm{o}} / \mu_{\mathrm{i}}$.

FIG. 8. The calculated ratio, $k=\mu_{\mathrm{o}} / \mu_{\mathrm{i}}$, as functions of $\mu_{\mathrm{i}}$ and $g_{\mathrm{syn}}$ for the time-independent ISI input to silent neurons: only integer $k$ 's are shown by circles $(k=1)$, squares $(k=2)$, triangles $(k=3)$ and diamond $(k=4)$, the cross denoting no outputs.

FIG. 9. Time courses of (a) input $U_{\mathrm{i}}$ and (b) output $U_{\mathrm{o}}$, and return maps of (c) input and (d) output ISIs for the sinusoidal modulation for $d_{0}=2 d_{1}=20 \mathrm{msec}$ (Eq.19).

FIG. 10. Time courses of (a) input $U_{\mathrm{i}}$ and (b) output $U_{\mathrm{o}}$, and return maps of (c) input and (d) output ISIs for the sinusoidal modulation for $d_{0}=2 d_{1}=10 \mathrm{msec}$ (Eq.19).

FIG. 11. Histograms of (a) input ISI with sinusoidal modulation and (b) output ISI. Solid (dashed) curves are for $d_{0}=2 d_{1}=10(20)$ msec. 
FIG. 12. Mean ( $\mu_{\mathrm{o}}$, solid curve) and rms $\left(\sigma_{\mathrm{o}}\right.$, dashed curve) values of output ISI against the mean value $\left(\mu_{\mathrm{i}}\right)$ of input ISI with sinusoidal modulation $\left(c_{\mathrm{vi}}=0.40\right)$. Filled circles denote the distribution of output ISIs for a given $\mu_{\mathrm{i}}$, dotted curves denoting $k \equiv \mu_{\mathrm{o}} / \mu_{\mathrm{i}}$.

FIG. 13. Time courses of (a) input $U_{\mathrm{i}}$ and (b) output $U_{\mathrm{o}}$, and return maps of (c) input and (d) output ISIs for chaotic inputs generated by the Rössler model (case R1).

FIG. 14. Histograms of (a) input ISI and (b) output ISI for the chaotic input generated by the Rössler model, and (c) output ISI for its surrogate, solid (dashed) curves being for case R1 (R2).

FIG. 15. Return maps of (a) input ISI and (b) output ISI for the chaotic input generated by the Rössler model (case R2); (c) and (d) are corresponding return maps of its surrogate data.

FIG. 16. Return maps of (a) input ISI and (b) output ISI for the chaotic input generated by the Lorentz model; (c) and (d) are corresponding return maps of its surrogate data.

FIG. 17. Histograms of (a) input ISI and (b) output ISI for spike-train inputs with the Gamma distribution; solid (dashed) curves for input ISIs of $\mu_{\mathrm{i}}=10(20)$ msec with $c_{\mathrm{vi}}=0.40$.

FIG. 18. Mean ( $\mu_{\mathrm{o}}$, solid curves), rms ( $\sigma_{\mathrm{o}}$, dashed curves) and $c_{v o}$ (thin solid curves) of output ISIs against the mean value $\left(\mu_{\mathrm{i}}\right)$ of input ISI for Gamma distribution with (a) $c_{\mathrm{vi}}=0.4$ and (b) $c_{\mathrm{vi}}$ $=1.0$. The dotted curves denoting $\mu_{\mathrm{o}}=\mu_{\mathrm{i}}$ are plotted for a guide of the eye

FIG. 19. $c_{\mathrm{vo}}$ against $c_{\mathrm{vi}}$ for inputs with time-independent ISI (inverted triangles, $c_{\mathrm{vi}}=0$ ), with sinusoidal modulation (open diamonds, squares and triangles for $c_{\mathrm{vi}}=0.22,0.40$ and 0.82 , respectively), and with random Gamma distribution (filled squares, triangles and inverted triangles for $c_{\mathrm{vi}}=0.40,0.75$ and 1.03 , respectively). The dotted curve expressing $c_{\mathrm{vo}}=c_{\mathrm{vi}}$ is plotted for a guide of the eye.

FIG. 20. Time courses of spike-train inputs and outputs; input ISIs are (a) $T_{i}=5$, (b) 10 and (c) $20 \mathrm{msec}$, and upper (lower) panel of each figure shows inputs (outputs). 
This figure "fig1-4.jpg" is available in "jpg" format from: http://arxiv.org/ps/cond-mat/9906020v3 
This figure "fig5-8.jpg" is available in "jpg" format from: http://arxiv.org/ps/cond-mat/9906020v3 
This figure "fig9-12.jpg" is available in "jpg" format from: http://arxiv.org/ps/cond-mat/9906020v3 
This figure "fig13-15.jpg" is available in "jpg" format from: http://arxiv.org/ps/cond-mat/9906020v3 
This figure "fig16-18.jpg" is available in "jpg" format from: http://arxiv.org/ps/cond-mat/9906020v3 
This figure "fig18-20.jpg" is available in "jpg" format from: http://arxiv.org/ps/cond-mat/9906020v3 